\title{
ROLES FAMILIARES Y MERCADO DE TRABAJO*
}

\author{
Xavier Coller i Porta \\ Universitat Autònoma de Barcelona
}

Resum

La diferent posició dels individus en el mercat de treball sembla convertir-se en una variable (no l'unica) amb un fort pes explicatiu de determinades pràctiques familiars que s'analitzen en aquest article. Aixi, les desigualtats viscudes a la familia per raó de sexe poden explicar-se per la desigual situació d'homes $i$ dones en l'esfera laboral; unes desigualtats a les quals donen suport discursos diferenciats que, a la vegada, determinen unes pràctiques socials que ens permeten distingir (en un pla teòric) tres models de familia: la tradicional-segregacionista, la rupturista-modernitzadora i la mixta.

Resumen

La posicion diferente de los individuos en el mercado de trabajo puede convertirse en una variable (no única) con fuerte peso explicativo de determinadas prácticas familiares que se analizan en este artículo. Así, Las desigualdades vividas en la familta por razón de sexo pueden explicarse por la desigual situación de bombres y mujeres en la esfera laboral; unas desigualdades que están respaldadas por discursos diferenciados que, a su vez, determinan unas prácticas sociales que nos permitirán distinguir (en un plano teórico) tres modelos de familia: la tradicional-segregacionista, la rupturista-modernizadora y la mixta.

Abstract

The different position of individuals in the labour market seems to become a variable - not unique, though-with heavy explanatory weight of specific family practices which are analysed in this article. Thus, the family inequalities are backed by differentiated reasoning powers, wich at the same time settle certain social practices that will alow us to distinguis -on a theoretical level-three patterns of family: the traditional-segregationist, the rupturist-modernizing and the mixed one.

* Este trabajo es un resumen de la Menioria de licenciatura presentada en 1989 cn la Facultat de Ciències Polítiques i Sociologia de la Universitat Autònoma de Barcelona. 
"Papers»: Revista de Sociologia

\section{INTRODUCCIÓN. PUNTOS DE PARTIDA}

En nuestra investigación hemos tratado de interconectar dos campos específicos de la sociología: trabajo y familia. Dos ámbitos que nos definen los dos espacios básicos en los que se desarrollan/socializan los individuos: el espacio público y el privado. Es importante remarcar que es en la familia primero y en el trabajo después donde los individuos configuran su personalidad a través de un proceso de socialización en el que, indudablemente, intervienen otros ámbitos (sistema educativo, medios de comunicación, grupos de referencia). Pero es en el mercado de trabajo y en el grupo familiar - los espacios público y privado por excelencia- donde los individuos pasan la mayor parte de su tiempo y, por tanto, donde el proceso de socialización (o resocializacióny se hace de forma más acentuada.

Lo público y lo privado se interconectan. Y su punto de relación es la familia, entendida, ésta, más que como un agregado de individuos, como un conjunto de relaciones entre seres humanos unidos por algún lazo de parcntesco o afectividad y que realizan alguna función en común. Es en la familia en la que se manifiestan las contradicciones que la sociedad genera entre los individuos, actuando el grupo familiar en algunos casos como reflejo de estas contradicciones y en otros como refugio ante las mismas.

Se trata de un ámbito, el privado, en el que la esfera pública penetra en distintas direcciones: ideológica, jurídica, religiosa, política, comunicativa... de tal forma que la evolución de la familia parece ser el refiejo de la propia sociedad. Cuando menos, sí que podemos afirmar que lo público afecta, moldea, hace variar lo privado, a pesar de que en este ámbito las resistencias a adoptar los cambios de la sociedad puedan ser intensas, provocando desajustes entre el plano ideológico y el pragmático (nos referimos a la contradicción que existe entre lo que se piensa -reflejo de la esfera pública penetrada por la ideología dominante-y lo que se hace en el ámbito privado). Una contradicción quae se manifiesta en la familia de una forma clara y contundente.

La sospecha de que estos dos espacios están interconectados apareció con el interrogante que daba origen a nuestra investigación: en el interior de la familia se viven desigualdades sexuales que tienen su reflejo en los roles que la sociedad reserva a los integrantes del grupo familiar. ¿Por qué?, ¿cuáles son los papeles que hombres y mujeres desempeñan en la familia?, ¿por qué?, ¿tiene esto alguna relación con la situación que se vive en el mercado de trabajo y, por tanto, con la obtención de ingresos? Si tiene relación, ¿̨en qué medida y de qué forma?, ¿cómo afecta la evolución del mercado de trabajo a la vida familiar? y, más en concreto, ¿cómo afecta a los roles que desempeñan hombres y mujeres? La respuesta a estos interrogantes iba a guiarnos a lo largo de nuestra investigación. 
Roles familiares y mercados de trabajo

\section{FAMILIA Y ROLES FAMILIARES}

Previamente se había hecho una revisión teórica de las principales hipótesis desarrolladas por investigadores - principalmente europeos y norteamericanos-que, siquiera colateralmente, habían tratado el tema de nuestra investigación. De esta revisión extrajimos los conceptos suficientes para construir el armazón teórico que sustentaba nuestra investigación. Parafraseando a Inés Alberdi definimos la familia como el conjunto de "dos o más personas unidas por el matrimonio o la filiación (por extensión la afectividad), que viven juntos, ponen sus recursos económicos en comín y consumen juntos una serie de bienes» (Alberdi, I., 1982: 90). La dimensión afectiva, la económica y la de consumo se nos antojaban como los elementos que mejor podrían caracterizar nuestras unidades de observación ${ }^{1}$.

La institución familiar ha supuesto en el seno de cada sociedad una determinada distribución de roles entre los hombres y las mujeres. Interesa detenernos momentáneamente en este aspecto para explicar que entenderemos por familia algo más que un agregado de individuos que comparten un hogar y realizan juntos una serie de funciones (consumo, producción, reproducción, etc.). Efectivamente, en el seno de la familia se reproducen un complejo de relaciones que configuran un sistema de roles interactivos que son desempeñados de forma distinta por los miembros que la componen. Dicha división de roles se manifiesta en el seno de la familia con la predominancia del varón y la supeditación económica del resto de los miembros. Se trata de una predominancia económica que obedece a su posición privilegiada en la estructura

1. A efectos de aclarar este punto señalaremos que en el trabajo original conceptualizamos al grupo familiar como una institución que se desarrolla tanto en la esfera pública como en la privada. De la primera extrae su legitimación individual, y en ambas desempeña unas funciones básicas.

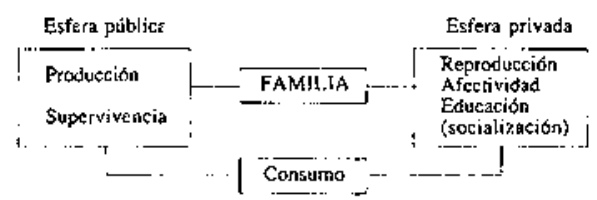

No obstante, aunque sean éstas las funciones básicas que desempeña la familia en la sociedad, no es menos cierto que otras instituciones sociales pueden realizar a la perfección alguna de ellas. Con ello estamos serialando que nuestra visión de la familia no es armónica, como pretende el funcionalismo, ya que las funciones que desar rolla la farnilia no son consideradas como una contribución al normal funcionamiento de la sociedad, sino como una resputesta a las exigencias de ésta. Al mismo tiempo, esta contribución a la sociedad (en la que radica su carácter funcional) puede ser igualmente desarrollada por otro tipo de instituciones (escuela, estado, etc.). 
productiva, en el mercado de trabajo (Michel, 1974 a, b; Edgell, 1980; Rapoport \& Rapoport, 1973). Diversos autores han puesto de manifiesto la relación existente entre la obtención de un salario y la asignación de un rol determinado en el seno de la familia, hecho que ya fue observado por Engels cuando señalaba que "la preponderancia del hombre en el matrimonio es consecuencia, sencillamente, de su preponderancia económica, y desaparecerá por si sola con ésta" (Engels, 1983: 151). Esta profecía, lejos de cumplirse, no hace sino servirnos de excusa para poner de manifiesto la actualidad de la realidad que engloba.

En resumen, adaptando la idea de Edgel1 ${ }^{2}$ (1980) podemos decir que la división sexual en el seno de la familia - y por tanto, la desigual asignación de roles en la misma - tiene una estrecha relación con el papel que se desempeña fuera de ellia, y mucho nos tememos que dicha división no obedece a causas biológicas (aunque tradicionalmente, y desde posturas legitimadoras de este statu quo, se ha intentado explicar con esta argumentación), sino que tiene origen en la desigual posición de los cónyuges en aquel ámbito que les permite obtener los medios de subsistencia. En nuestra sociedad neocapitalista, postindustrial, de capitalismo avanzado, o como quiera llamársela, dicho ámbito se centra en el mercado de trabajo. Será, pues, la desigual ubicación en un mercado laboral con una fuerte tendencia a la segmentación y precarización, la que produzca -y ésta es nuestra hipótesis principal-, una progresiva y acusada tendencia a la asimetría de roles en el seno de la familia, que tiene como expresión más clara el hecho de que mientras la mujer se incorpora al mercado de trabajo, el varón no asume más responsabilidades en el reparto de tareas domésticas, y cuando lo hace es de una forma dependiente (Izquierdo, 1988).

\section{EL MERCADO DE TRABAJO}

Vistas así las cosas parecía pertinente realizar un somero análisis del mercado laboral español para descubrir posibles tendencias que nos permitieran aventurar alguna hipótesis útil para nuestro estudio.

El primer elemento que cabe reseñar aquí es la creciente precarización del mercado de trabajo. Una precarización que ha acompañado a la flexibilización laboral que tantos ríos de tinta ha hecho verter a sindicatos, empresarios y gobierno. Efectivamente, con el objetivo principal de crear puestos de trabajo se

2. Edgcll localiza la desigualdad conyugal en la dependencia del marido respecto de su empleo, y la dependencia de la fanilia respecto del marido para el sustento financiero y, por ende, la dependencia de la esposa respecto del marido. 
promulgan una serie de decretos-ley cuyo objetivo era ampliar las modalidades de contratación (Ministerio de Trabajo y Seguridad Social, 1988), favoreciendo y fomentando el empleo temporal frente a los contratos fijos. Paralelamente, y desde 1976, con la derogación de una cláusula de una ley del primer gobierno de la monarquía, se reducían los costes del despido laboral y se ampliaban las posibilidades de recurrir a él. Este doble proceso flexibilizador lleva aparejada una mayor degradación del factor trabajo. Y por degradación entendemos una mayor inestabilidad laboral; una progresiva segmentación de los trabajadores que, colectivamente, se estructuran en torno a un núcleo central con trabajo estable y una amplia parcela flexible caracterizada por la precariedad (Piore, 1983); pérdida de capacidad de acción de los sindicatos que ven cómo su medio natural de desarrollo (los trabajadores) se desestructura en virtud de nuevas modalidades de contrato que hacen que el trabajador sea fácilmente sustituible (con unos costos relativamente bajos o gratuitos para el empresario); una dificultad cada vez mayor para crear expectativas de vida si entendemos que éstas van ligadas a la independencia económica que facilitan unos ingresos continuados y estables. La estrategia seguida en todo este proceso ha consistido en "mantener como mano de obra estable en la empresa sólo un núcleo de asalariados que aseguren las actividades esenciales, recurriendo a los contratos precarios y a la subcontratación para el resto de tareas, haciendo de este último un factor de producción que pueda comprimir a su gusto (el empresario). Ello comporta la segmentación del mercado de trabajo y hace que los trabajadores de esta segunda zona, mal protegidos, soporten todo el peso de la adaptación de la empresa» (Zufiaur, 1986).

De resultas de todo ello podemos concluir que con el objetivo de fomentar la ocupación (2.970.000 parados en el primer trimestre de 1985), muy afectada por las crisis del 73 , se intenta flexibilizar el mercado laboral siguiendo las recomendaciones de la $\mathrm{OCDE}^{3}$. La consecuencia de todo elio es una mayor de-

3. La OCDE elabora un estudio en el que se concluye que aunque la flexibilidad del mercado de trabajo no es la solución ideal para el grave problema que entrañan las altas tasas de paro originadas por la crisis y el ajuste económico, tampoco supone una amenaza para la seguridad laboral. Sus autores distinguen seis areas de aplicación de los mecanismos flexibilizadores (MTSS, 1986):

1. Los costes salariales, buscando en esta línea políticas permanentes de moderación salarial.

2. Las condiciones de empleo, buscando el contrato temporal frente al indefinido, explotando al máximo las posibilidades de los contratos a tiempo parcial.

3. La movilidad laboral, tanto en sentido funcional (de un puesto a otro) como geográfico.

4. La normativa jurídico-laboral, cuya excesiva rigidez enticnden que encorseta e impide el desarrollo de las relaciones laborales.

5. La organización del trabajo, tratando de establecer nuevos esquemas de trabajo, horarios, reducción de jornada, etc.

6. La formación profesional. 
"Papers»: Revista de Sociologia

gradación en el empleo a través de modalidades de trabajo que afectan de forma desigual a hombres y a mujeres y que ${ }^{\prime . . .}$ son hasta el momento y de ordinario formas precarias o inestables y devaluadas, pobres o subprotegidas, que sirven a las cambiantes necesidades empresariales para adecuar la mano de obra ocupada a las fluctuaciones de la producción y del mercado en un clima sociocultural especialmente sensible a los valores empresariales y de la economía de mercado»(Casas, 1989).

La segunda cuestión que es preciso resaltar es que hombres y mujeres se insertan en el circuito de trabajo de forma diferente. Ya de entrada se marcan enormes diferencias en cuanto a la cantidad de personas de uno u otro sexo que se declaran activas. De esta forma, no nos debe de extrañar que el porcentaje de mujeres activas bascule en torno al $30 \%$ mientras que el de los hombres se mueva alrededor del $70 \%$. No obstante, a partir de 1981, y más en firme desde $1985^{4}$, la tasa de actividad masculina comienza a descender suavementc y la femenina se incrementa hasta sobrepasar la barrera del $30 \%$ (Gráfico 1). La explicación a esta situación la encontramos en el gráfico 2, donde se puede observar que el incremento de población femenina en el mercado de trabajo es muy superior al de los hombres. Esta entrada masiva de mujeres en el mundo laboral durante el periodo analizado merece una explicación. Desde el lado de la oferta, las causas que provocan el incremento de las tasas de actividad femeninas las podemos dividir en cuatro:

Ritmo de crecimiento de la población activa según sexo. 1977-1988. (Fuente: Elaboración propia a partir de los datos de la EPA, 1er. trimestre de las series anuales).

a) Demográficas: La reducción de las tasas de fecundidad desde mediados de los sesenta y la incorporación al mercado de trabajo, en la década de los 80 , de las generaciones del "baby boom", son las dos razones básicas que explican en gran medida el incremento de las tasas de actividad femenina, sobre todo desde mediados de los 80 .

b) Nivel de instrucción: Diversos estudios en páses europeos y en cl nuestro han puesto de manifiesto que cuanto mayor es el nivel académico obtenido por las mujeres más elevada es la tasa de actividad. No es de extrañar, pues, que como señalan Alabart y Solsona, la población femenina invierta en educación para posteriormente insertarse en el mercado laboral con ciertas aspiraciones de cualificación (Alabart \& Solsona, 1987)

c) Condiciones económicas: La situación económica individual o familiar hace que muchas mujeres decidan buscar un trabajo para aumentar los ingre-

4. Es de señalar que esta fecha es tomada por algunos economistas como el inicio de la recuperación económica tras la crisis del 73 y la más dura todavía del 78. 
sos necesarios para la subsistencia. Ello hace que se declaren activas (generalmente buscadoras del primer empleo), aunque otra cosa muy distinta es que consigan encontrar la ocupación deseada.

d) Condiciones sociales: Cada vez son más numerosas las mujeres que aspiran a la independencia que supone tener un empleo remunerado. A este hecho se une la tendencia creciente a la creación de familias monoparentales en las que la mujer se hace cargo de los hijos/as (Flaquer \&. Soler, 1988) y para ello debe disponer de una renta suficiente.

Desde el lado de la demanda se ha de decir que a pesar de que en épocas de crisis la ocupación femenina es recesiva, desde el año 1985 la demanda de mano de obra femenina crece en un proceso paralelo al crecimiento del sector

\section{Gráfico 1}

Tasa de actividad según el sexo. 1977-1988. (Fuente: Elaboración propia a partir de los datos de la EPA, 1er. trimestre de las series anuales).

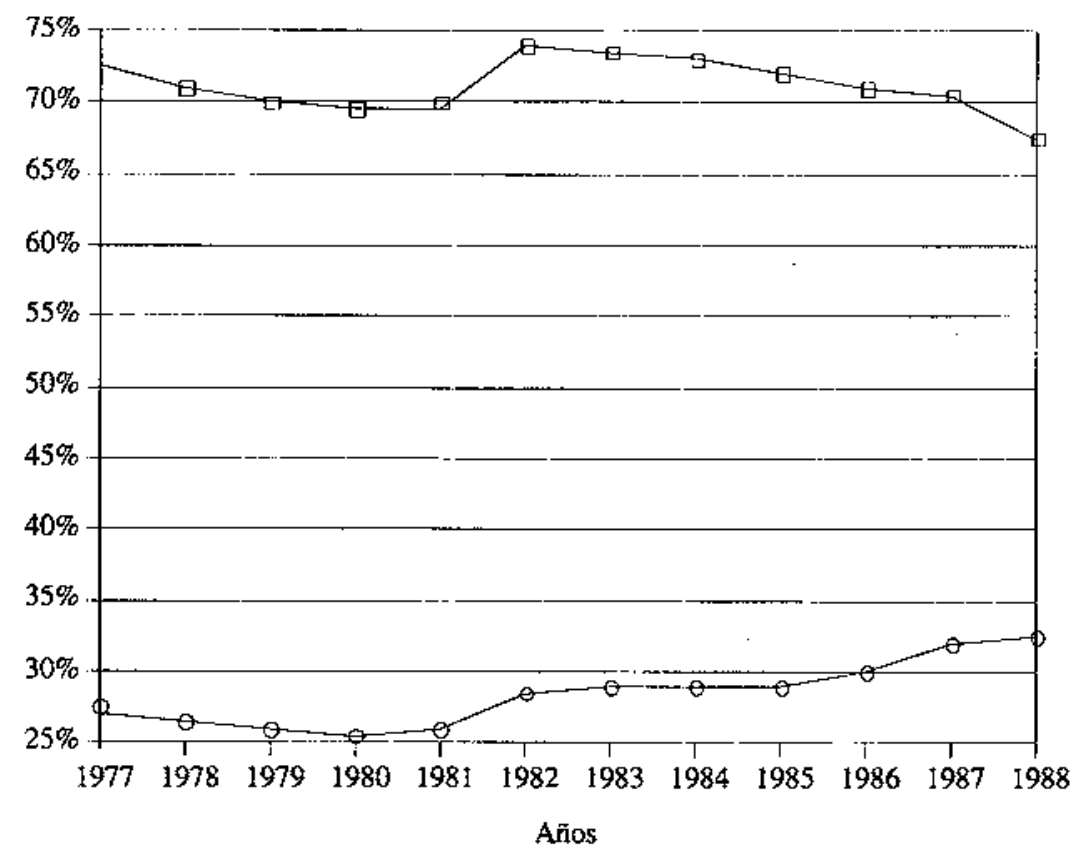

$\square$ Hombres $\quad$ Mujeres 
«Papers»: Revista de Sociologia

de los servicios y de la Administración Pública. Al mismo tiempo, la ampliación del abanico de las contrataciones pone sobre la mesa la posibilidad de que las mujeres accedan a empleos que pueden compaginar con las cargas familiares de las que se hacen responsables. (El hecho de que se hagan responsables no será analizado aquí, pero tiene relación con los papeles que tradicionalmente se ha reservado para la mujer en la familia).

Si bien esta incorporación puede ser calificada de espectacular (Gráfico 2), no es menos cierto que, una vez ubicada en el mercado laboral, la mano de obra femenina está destinada las más de las veces al paro. En términos absolutos la ocupación (hombres y mujeres) desciende en el estado español al pa-

\section{Gráfico 2}

Ritmo de crecimiento de la población activa según sexo. 1977-1988. (Fuente: elaboración propia a partir de los datos de la EPA, 1er. trimestre de las series antales).

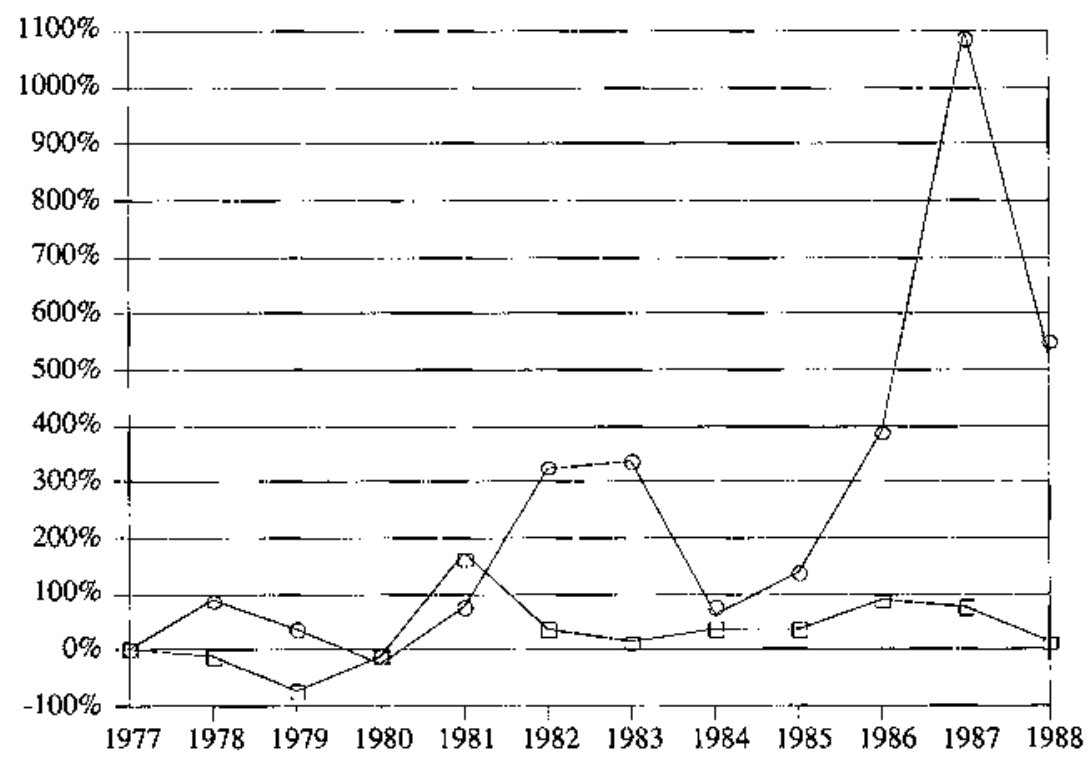

Años

$\square$ Hombres

O Mujeres 
sar de más de 12 millones de empleados a 11,5 millones en 1988. Dicho descenso es progresivo hasta 1985, año en que parece recuperarse lentamente el empleo. Ahora bien, la tendencia más acusada a la pérdida de ocupación ${ }^{5}$ la vive la población femenina, tal y como puede verse en los gráficos 3 y 4.

\section{GRAFICO 3}

Tasa de ocupación según sexo. 1977-1988. (Fuente: Elaboración propia a partir de los datos de la EPA, ler. trimestre de las series anuales).

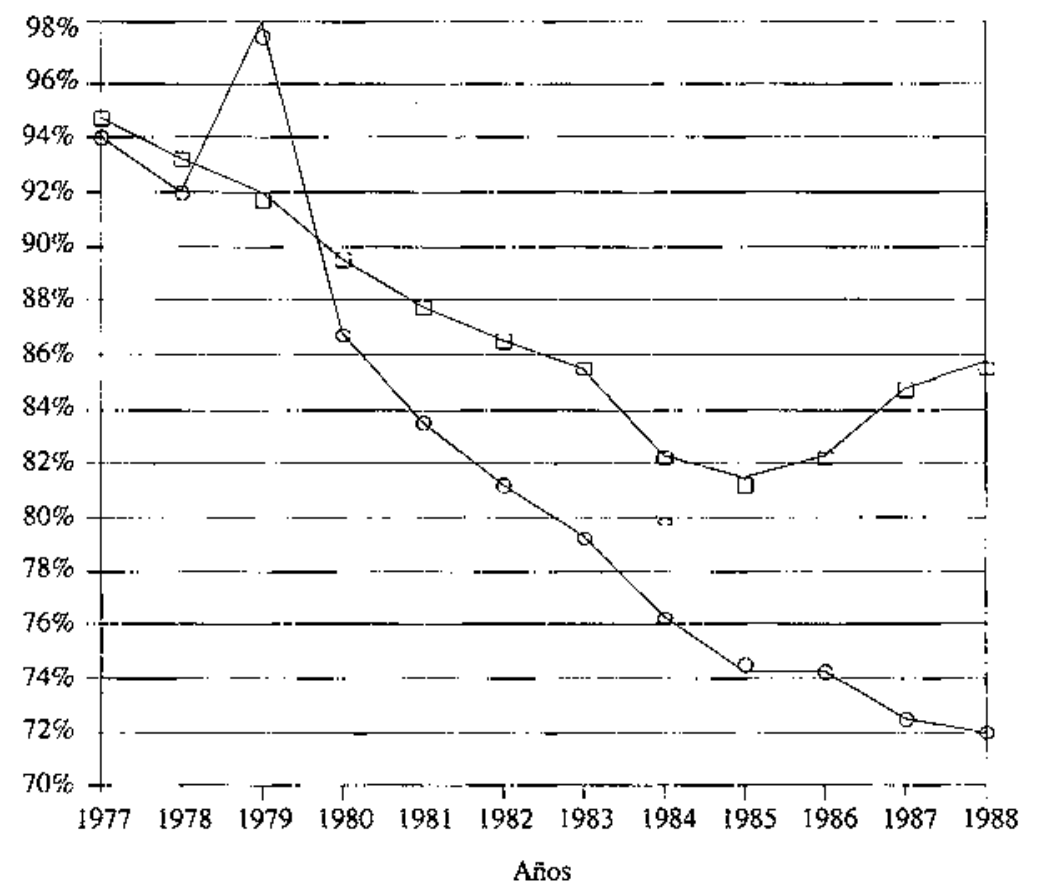

[) Hombres O Mujeres

5. Nötese que estamos trabajando con tasas de ocupación y paro, las cuales están sometidas a las variaciones de la población activa tocal. Es cierto que en términos absolutos la población ocupada femenina sufre una variación negativa $(-12,29 \%)$ inferior a la masculina $(-14,16 \%)$ durante el período estudiado. 
«Papers»: Revista de Sociologia

De resultas de nuestros análisis cabe decir que aunque la mujer se incorpora masivamente al mercado de trabajo cuando la coyuntura económica es favorable, no es menos cierto que dicha incorporación se hace engrosando la categoría de desempleados en las estadísticas oficiales.

Para no hacer excesivamente largo este apartado esbozaremos algunos puntos a modo de conclusión de los datos que recopilamos para nuestro estudio.

1. El mercado de trabajo prima con el empleo al varón casado, penalizando a los varones solteros y a las mujeres casadas (ver gráficos 5 y 6). Esto se ve más fácilmente si seccionamos el paro según el sexo y el estado civil: el desempleo se incrementa entre los solteros en un $296,7 \%$ y entre las casadas en un $¡ 1.450 \%$ !, mientras que entre los casados sólo se incrementa en un

\section{Gráflco 4}

Tasa de paro según sexo. 1977-1988. (Fuente: Elaboración propia a partir de los datos de la EPA, 1er. trimestre de las series anuales).

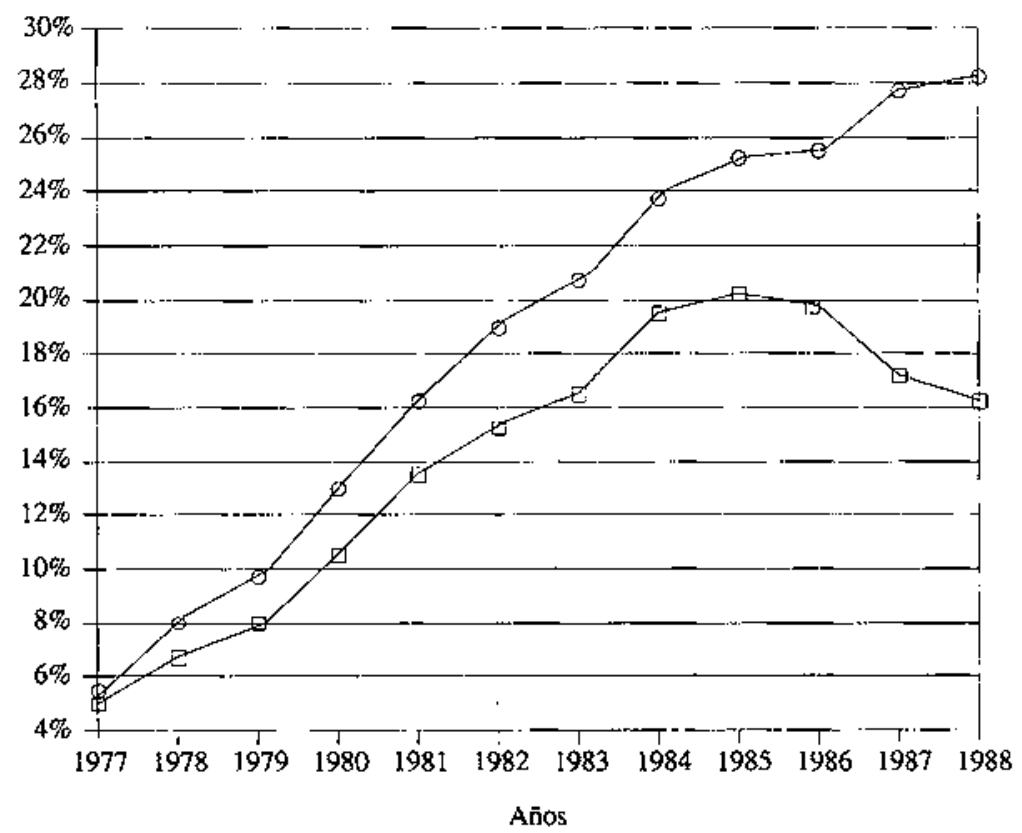

$\square$ Hombres o Mujeres 
$63,7 \%$ durante el período estudiado. Ello pone de manifiesto que las «invisibles" leyes del mercado se orientan a proteger laboralmente a un sector muy determinado de la población, precisamente a aquel sector que la tradición identifica con los cabezas de familia, los varones casados. Y, al mismo tiempo, se puede colegir que otro sector no menos importante numéricamente, el de las esposas, se margina o precariza en el trabajo (vía paro, vía contratos precarios) quizá porque esta misma tradición convertida en ideología dominante legitima el trabajo del varón casado pero apenas considera el trabajo de su esposa en la esfera pública, y cuando lo hacc adquiere el rango de «ayuda familiar».

2. La mujer casada sc inserta en el mercado de trabajo activo a través del paro, no de la ocupación. Ello influye para que muchas de ellas retornen a la categoría de desanimadas. Pero la sola declaración de actividad por parte de la mujer, sea casada o soltera, pone de manifiesto la voluntad de ésta por trabajar y, aunque sólo sea en el plano de las mentalidades, esto es significativo de que al menos la mujer comienza a liberarse de ciertos corsés que la cons-

\section{GRÁlilCO 5}

Tasa de ocupación por estado civil. Varones. 1977-1988. (Fuente: Elaboración propia a partir de los datos de la EPA,

1er. trimestre de las series anuales).

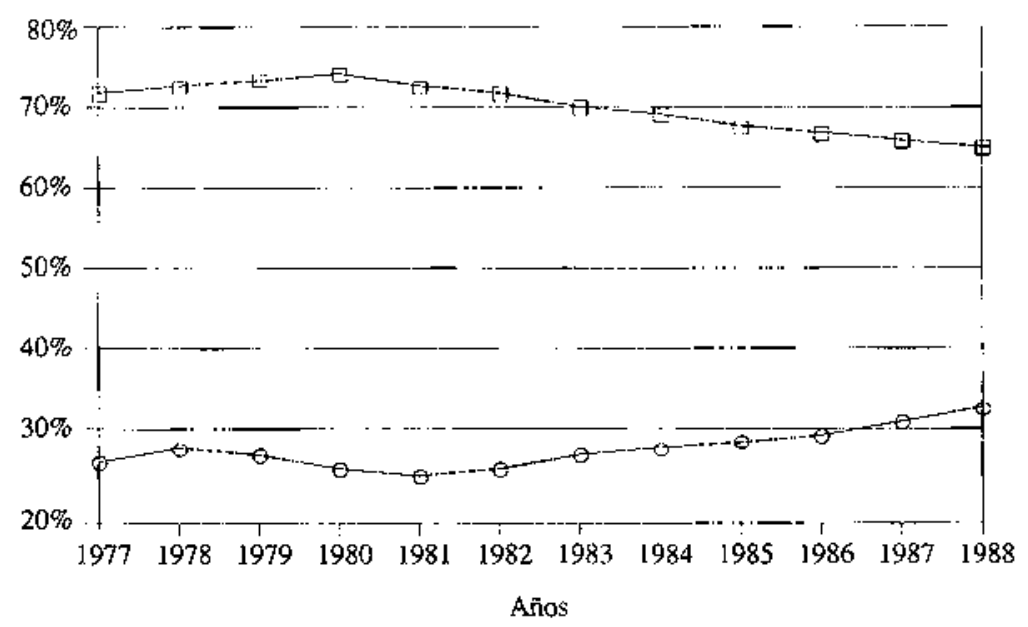

$\square$ Casados O Solteros 
«Papers»: Revista de Sociologia

treñian a las tareas del hogar. Otra cosa muy diferente es que el mercado de trabajo le permita librarse realmente de estos corsés a través de un empleo digno y en igualdad de condiciones que sus compañeros varones.

3. El distinto ritmo de crecimiento del paro, pero también de la ocupación y la actividad, no hacen más que reflejar que la mujer constituye una mano de obra generaimente barata, pero muy flexible, susceptible de ser utilizada o despreciada en función de los vientos que soplen en la economía. A tal punto es así que «estas fuertes variaciones dentro de la situación de la mujer ante el trabajo sugieren que ésta se inserta o no en el mundo laboral precisamente porque se ve empujada a rellenar huecos ocasionales que surgen en el mercado de trabajo o a actuar como mano de obra subsidiaria y de-

\section{GRAFICO 6}

Ocupados segúni estado civil y sexo. Casados. 1977-1988. (Fuente: Elaboración propia a partir de los datos de la EPA, 1er. trimestre de las series anuales).

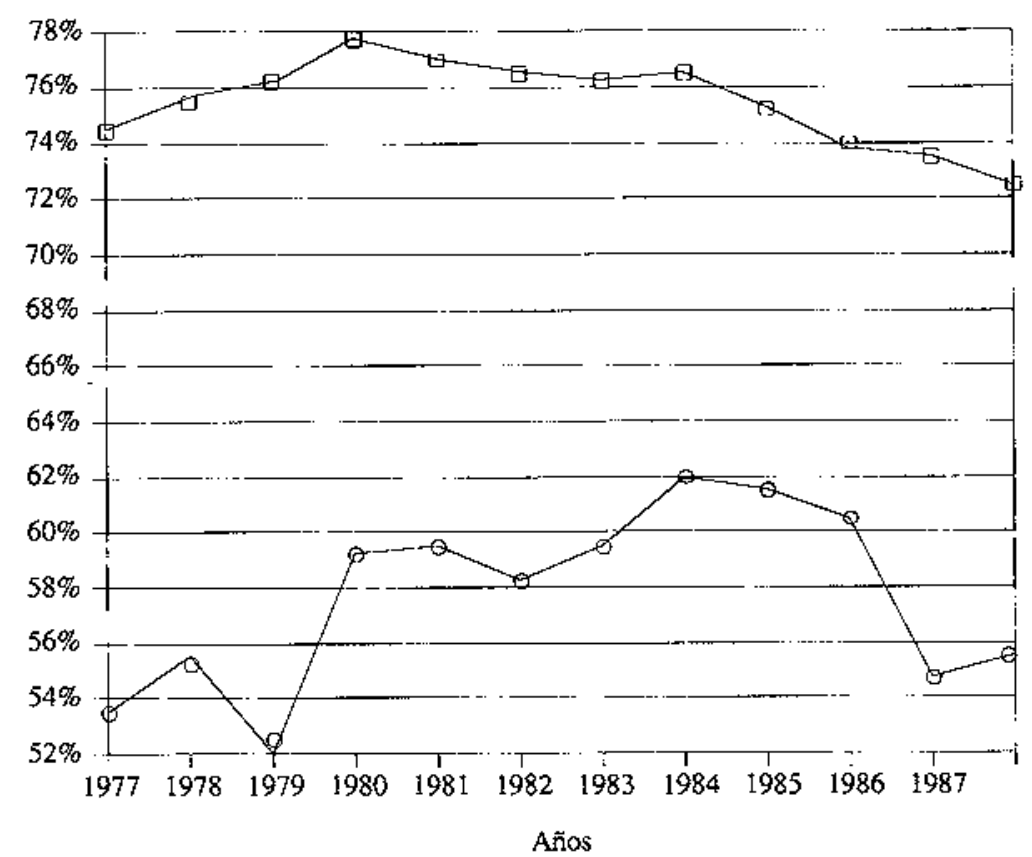

[) Hombres 0 Mujeres 
pendiente de la mano de obra masculina. Existirían, así, mecanismos socioeconómicos que en distintos momentos de la evolución histórica producen "nichos" a los que van destinadas las mujeres dentro del mercado de trabajo» (Casas, 1987: 141).

Cuando estos nichos son insuficientes se produce el incremento del desempleo femenino o la simple no participación laboral de la mujer, es decir, su vuelta a las actividades domésticas. Hay que decir que estos mecanismos socioeconómicos de los que habla J.I. Casas no son otros que la crisis económica y la práctica social habitual de favorecer laboralmente al varón casado en detrimento de la mujer.

\section{HIPÓTESIS DE TRABAJO NO FORMALIZADAS}

Llegados a este punto, hecha una revisión de textos y un análisis de las recientes tendencias del mercado laboral, estábamos en disposición de elaborar una serie de hipótesis de trabajo (no formalizadas) que en el trabajo de campo nos encargaríamos de validar o refutar. Trataremos de rcsumirlas en breves líneas.

Hombres y mujeres se introducen de forma diferente en el circuito laboral. A ellos se les reserva el trabajo que configura los segmentos centrales; a ellas se las desplaza hacia la periferia. ¿Por qué?, ¿qué consecuencias tiene? Este proceso, resultado de una dinámica laboral a la que no se escapan ni empresarios, ni gobierno ni sindicatos, permite hacer dos consideraciones:

a) El porqué. En una sociedad patriarcal la figura (rol) del cabeza de familia tiene dos características básicas:

1. Se asocia a los individuos de sexo masculino.

2. Es objeto de protección laboral, sea directamente por la legislación o los acuerdos colectivos, sea indirectamente por la práctica habitual de despedir, en caso de crisis, a las mujeres y a los jóvenes que trabajan, antes que a los adultos varones, o por contratar preferentemente a los hombres que a las mujeres.

Ello, creemos, es consecuencia de la ideología/mentalidad dominante en nuestra sociedad: el varón a procurar el sustento económico, y la mujer a dedicarse al hogar y la crianza de los hijos/as. Bien es cierto que desde nuestro punto de vista ambas funciones son consideradas dentro del eje producción/reproducción con el que habíamos conceptualizado a la familia, entendiendo que el trabajo del hogar tiene una indiscutible faceta productiva más similar al empleo del sector servicios que al de la construcción o la indus- 
tria. No obstante, lo que es importante señalar aquí es que esta visión determinada del grupo familiar sustenta y legitima prácticas sociales que, en el ámbito del espacio público, quedan manifiestas en la protección del varón-cabeza-de-familia. Esta visión tradicional, además, tiende a valorar negativamente el trabajo de la mujer casada, o cuando menos a considerarlo de forma marginal como una «ayuda» a los ingresos regulares que aporta el varón a la familia (A labart et al., 1988).

b) Las consecuencias. Si nuestra hipótesis principal tiene visos de ser real, la progresiva incorporación de la mujer al mercado de trabajo y, por tanto, su aportación ${ }^{6}$ de ingresos a la familia, se debe corresponder con dos situaciones complementarias:

1. Una mayor participación del varón cabeza de familia en las tareas domésticas, debido a que ambos cónyuges pasan parte de su ticmpo fuera del hogar y es de esperar una redistribución equitativa de las tareas.

2. El acceso ${ }^{7}$ de la mujer a ámbitos de decisión y poder familiar reservados tradicionalmente al hombre por desempeñar el papel central (aportación de ingresos) en el seno del grupo. Este acceso irá aparejado a una mayor consideración individual de la mujer en el seno de la familia.

Estas dos situaciones, a nuestro entender, son básicas para dilucidar y comprender el proceso de simetrización (igualación de roles) en la familia, a tal punto que cuanto más se dé «1» $\mathrm{y}$ «2», mayor democracia interna habrá en el grupo familiar. No obstante, hay que señalar que en el proceso de simetri-

6. En este punto hay que hacer una importante distinción: creemos que el proceso de simerrización al que más adelante hacemos referencia acontece cuando la cantidad y regularidad de los ingresos que aporta la mujer a la familia se hace en similares condiciones a las del hombre. Es decir, que lo que aporta la mujer no es considerado como una "ayuda familiar", como complemento, sino como una fuente más de ingresos. A partir de ahora, cuando hagamos referencia a la aportación económica de la mujer se deberá entender que dicha aportación se hace en similares condiciones a las del varón.

7. El acceso al que hacemos referencia debe ser considerado desde el puno de vista que exponemos a continuación: la ideología tradicional dominante reserva al varón una serie de funciones en la familia. Entre ellas destaca la autoridad y el poder de decisión. Creemos que ello es efecto del papel preponderante que le confiere su cualidad de "alimentador económico" del grupo familiar. Pues bien, lo que plantea esta hipótesis es la siguiente situación; si la mujer aporta unos ingresos en similares condiciones que el hombre (cuantia y regularidad), y éstos son considerados no como "ayuda familiar", sino como fuente real de ingresos, probablemente, el siguiente paso sería que la mujer accediera a cotas de poder similares a las que posee el varón en el grupo familiar que hemos definido. 
zación intervienen otras variables que nosotros no hemos considerado aquí por entender que en la raíz de este proceso la mujer juega un papel fundamental, adquiriendo mayores cotas de protagonismo. Ello significa conceder prioridad en el análisis a la situación de ésta en el mercado de trabajo, ya que será la posición en el circuito laboral la que determine el nivel y la regularidad de sus ingresos e, indirectamente, su grado de intervención en la esfera familiar. Sin embargo, dicha relación no debe considerarse desde un punto mecanicista, ya que una de las conclusiones a las que se ha llegado con nuestra investigación es que el hecho de que la mujer disponga de un salario no es condición suficiente para que se produzca una mayor intervención en las esferas de poder y decisión familiar, ni una mayor repartición de las tareas domésticas. En este proceso juegan un papel fundamental los modelos de socialización de los individuos.

Todo ello nos lleva a pensar que si en el mercado de trabajo existen desigualdades en función de variables como la edad y el sexo, la familia no escapa a este proceso. Es más, creemos que en el grupo familiar la división sexual del trabajo no está legitimada por elementos externos como en el mercado de trabajo (empleadores, por ejemplo), sino por aquello que hemos considerado la mentalidad tradicional dominante. ¿A qué nos referimos cuando empleamos este término? Sencillamente, al conjunto de percepciones y representaciones sociales construidas y reconstruidas por los individuos en función de los modelos en los que han sido socializados ${ }^{8}$.

Las desigualdades sextrales se ponen de manifiesto tanto en la esfera pública como en la privada, tanto en el mercado de trabajo como en la familia. Lo que no está tan claro es dónde comienzan a producirse y cómo se legitiman. No es de recibo señalar que en este proceso los dos ámbitos de actuación social que hemos definido están interconectados, de tal forma que ciertas variaciones en el mercado de trabajo acarrean consecuencias en la vida familiar: la disminución de la jornada laboral, la incorporación de la mujer y los hijos al trabajo, el desempleo, los niveles salariales, las modalidades de contratación, etc., configuran el elenco de variables públicas en torno a las que se estructuran los ritmos de vida privados.

8. Es rradicional porque es producto de la evolución de la sociedad y arranca de una visión determinada de los roles familiares que tiene una continuidad y agudización en la sociedad capitalista configurada tras la revolución industrialYY es dominante en un doble sentido: porque según han revelado diferentes estudios se trata de una mentalidad profusamente extendida (Michcl, 1974) que se refleja en la vida cotidiana familiar (división del trabajo doméstico, por ejemplo) y porque se trata de un conjunto de percepciones típicas de los grupos socialmente dominantes que ha iniernalizado y hecho suyas el resio de la sociedad (la ideologia de las clases dominantes se convierte en la idcologia dominante). 
Al mismo tiempo, tanto lo privado como lo público se hallan determinados por una forma de pensar, por una lógica social que hemos denominado ideología o mentalidad dominante. Ahora bien, ¿no sería más propio hablar de diferentes lógicas, de distintas mentalidades? Cuando hablamos de lo dominante no estamos asegurando su unicidad, sino su preponderancia, con lo cual señalamos que junto a esta mentalidad dominante en nuestra sociedad postcapitalista se han desarrollado otras lógicas, otros discursos que respaldan prácticas sociales diferentes a las habituales?

Lo que está por ver es la forma en que estas lógicas, cstos discursos, estas prácticas sociales familiares están o no influidas por variables como el tipo de trabajo que se desempeña, los ingresos, la disponibilidad de tiempo para dedicar al trabajo doméstico, el hecho de tener o no tener hijos, el nivel de estudios, etc.

La mentalidad tradicional y dominante a la que hacemos referencia tiene su más vivo reflejo en la forma de articulación familiar. Habíamos dicho que era la fuente legitimadora de la división sexual del trabajo en la esfera doméstica y la que orientaba la división de roles en la familia en virtud de un discurso y una práctica social profusamente extendida que asignaba al varón el papel de procurador de los ingresos y a la mujer el de reproductora (social -educación y crianza de los niños/as- y biológica). Por otra parte se había comentado que frente a esta lógica social existen otras prácticas que, a partir de ahora, denominaremos rupturistas o modernizadoras.

Pues bien, lo que hemos tratado de analizar en este estudio es cómo se articulan estas prácticas familiares, qué elementos las componen y las diferencian, qué discursos las respaldan, qué individuos emiten estos discursos y cuáles son sus características y elementos comunes. Todo ello con el objetivo de construir unos modelos (tipos ideales, en el sentido weberiano del término) que nos permitan identificar diversos grupos familiares en función de:

a) características externas a la familia (ubicación en el mercado de trabajo, tipo de empleo e ingresos, disponibilidad de tiempo, etc.) y

b) características internas (distribución de las tareas domésticas, definición

9. Tal es el caso, por ejemplo, de las parejas de cohabitantes (consideradas aquí como núcleos familiares aunque entre ellos no mcdie contrato matrimonial). En un interesante estudio, Alabart, Cabré y otros (1988) señalan que este tipo de fanilias manifiestan unos comportamientos diferenciados del resto: las tareas domésticas se reparten en función del tiempo disponible y de las obligaciones públicas de la pareja, y no en virtud del sexo al que cstén adscritos; la independencia de los individuos es un valor de alta consideración; la integración en la esfera privada no se hace por la vía de la dependencia y la subsidiariedad de unos miembros con respecto a otros. 
de los espacios de poder y decisión, administración del presupuesto familiar, división de roles, en suma) ${ }^{10}$.

\section{CONCLUSIONES MÁS SIGNIFICATIVAS}

Elaborado un marco teórico amplio, un sistema de hipótesis provisionales, hecho el trabajo de campo, nos proponíamos, en la última fase de nuestra investigación, elaborar un sistema de conclusiones provisionales que dieran sentido al trabajo que habiamos realizado. En las páginas que siguen trataremos de reconstruir someramente las claves de los discursos que elaboraron nuestros entrevistados para intentar resumir los modelos ideales que aportábamos como colofón de nuestra investigación. Partiendo de las hipótesis que han formulado otros sociólogos/as y de las que hemos apuntado nosotros, dichos modelos nos iban a permitir:

1. Identificar la bomologia de estructuras discursivas que existen entre las diferentes categorias familiares " que habiamos establecido.

2. Agrupar dichas familias en los modelos construidos en función de sus semejanzas discursivas.

3. Identificar las similitudes externas (variables exógenas) de las familias incluidas en cada uno de los modelos establecidos.

10. A modo ilustrativo diremos que la metodología que utilizamos para nuestro estudio se centró en entrevistas en profundidad reforzadas por la observación participante. Tanto en las entrevistas como en la observación se prestó atención a los siguientes ítems:

1. Plano de la praxis.

1.1. Lo manifiesto.

1.1.1. Distribución de tareas domésticas.

1.1.2 Ingresos y administración del presupuesto.

1.1.3. Uso del espacio.

1.1.4. Uso del tiempo de ocio.

1.2. Lo latente.

1.2.1. Ambitos de decisión.

1.2.2. Tispacios de poder.

2. Plano del discurso.

2.1. Visión de «su" familia.

2.2. Visión de los roles del hombre y la mujer en la familia.

2.3. Consideración del trabajo remunerado de la mujer.

2.4. Opinión sobre la igualdad de roles entre hombre y mujer en la familia.

11. Las familias que cstudiamos durante nuestro trabajo de campo las dividimos en tres grandes grupos: precarias, mixtas y estables, en función de la situación laboral en la que se cncontraran los miembros que aportaban ingresos económicos al grupo familiar. 
"Papers»: Revista de Sociologia

\section{TRES DISCURSOS, TRES MODELOS}

Una primera aproximación a los discursos que articulan nuestras unidades de observación nos permite distinguir tres modelos: uno marcadamente tradicionalista, otro moderadamente rupturista, y tno más que podemos considerar intermedio, ya que conticne elementos entremezclados de los dos anteriores. Antes de proseguir en la caracterización de éstos hay que hacer varias aclaraciones:

1. Los modelos que aquí presentamos son teóricos, aunque están basados en la observación que hemos realizado en nuestro trabajo de campo. El procedimiento seguido para su construcción ha consistido en abstraer de la realidad las características principales que conforman estos modelos y darles un contenido teórico.

2. Se trata de modelos ideales, a pesar de que su plasmación en la realidad concreta los haga desvirtuarse ligeramente.

3. Su validez es provisional, a expensas de ser contrastados por otras investigaciones.

Considerando estas tres premisas pasaremos a caracterizar de forma sintética los modelos que hemos construido.

\section{TRADICIONAL-SEGREGACIONISTA}

Este modelo se centra en un tipo de discurso cuya argumentación es la siguiente: biológicamente, hombres y mujeres son diferentes, y esta diferencia tiene un claro reflejo en la esfera social. La mujer, el sexo débil, tiene asignadas una serie de funciones cuya justificación radica en la continua apelación a la tradición. Será considerado "normal" que la mujer casada no trabaje (inactividad) y por tanto cuide del hogar, del esposo y de los hijos. Esta ideología de la diferencia tiene su aplicación en el ámbito familiar: si es la esposa la encargada de la reproducción (biológica y social), el varón no deberá inmiscuirse en competencias que no le son propias. Resultado de todo elio es que en la familia se asistirá a una segregación de los roles masculinos y femeninos tanto más acusada cuanto mayor sea el grado de convencimiento de los cónyuges de que csta situación es la "normal» y la deseable (bien sea porque desconocen otras formas de articular la familia, bien sea porque han decidido conscientemente construir así su vida familiar).

La mujer asume las tareas domésticas y el varón invierte su tiempo en el mercado de trabajo. Se convierte así en la única fuente de ingresos de la fami- 
Lia. Esto supone una fuerte dependencia de la mujer con respecto a su esposo o-adicionalmente-a a sus hijos si éstos trabajan. Pero dicha dependencia no es manifiesta sino latente, oculta tras una cortina de aparente «normalidad». Ya lo habíamos dicho: es «normal» que esto ocurra así y no de otra forma. El varón, constituido y legitimado socialmente como el sustento económico de la farnilia ejerce tal cualidad en dos ámbitos diferentes: la posibilidad de decidir y la de ejercer el poder que le confiere su posición central en la familia.

Efectivamente, el modelo segregacionista basa su razón de ser en una figura principal revestida de una autoridad que impone a los demás miembros de la familia (que sea por la fuerza o por persuasión es otra cuestión). Podemos hablar, pues, de un autoritarismo centralizado y de un poder demarcado. El esposo se convierte en árbitro, juez en caso de conflicto donde no es parte, una autoridad cuyas sentencias adquieren rango de ley para el resto de miembros de la familia. Será él quien decida sobre las cuestiones «importantes» de la vida familiar aunque en multitud de ocasiones sea la mujer la que le incite a tomar la iniciativa. El poder familiar, puesto en práctica en situaciones de conflicto, radica en esa centralidad autoritaria.

Se trata de un modelo cuyo reflejo en la práctica social habitual pasa por un triple desequilibrio: financiero, de distribución de tareas domésticas, y de reparto de poder y autoridad. Dichos desequilibrios presentan una estrecha relación precisamente con el papel subsidiario que posee la mujer en las familias articuladas de este modo. Y esta subsidiariedad es originada por su dependencia total del marido en lo económico. Bien es cierto que en ocasiones -esto lo veremos más adelante-- se da el caso de que la mujer trabaja fuera del hogar y aporta unos ingresos $y$, sin embargo, continúa presentando una fuerte dependencia. A modo de hipótesis habría que hablar de la diferente consideración que se tiene de estos ingresos, ya sean entendidos como una ayuda al sueldo del varón o como una fuente financiera más, autónoma e independiente.

Un último trazo nos permitirá caracterizar este modelo: sus miembros poseen una red social densa (Bott, 1981; Kellerhals, 1984) que, a la postre, será común. Es decir, los círculos de amistades con los que se comparte el tiempo de ocio son comunes a uno y otro cónyuge, de tal forma que en ellos se encuentra una fuente de legitimación y justificación del propio modelo familiar.

\section{RUPTURISTA-MODERNIZADOR}

Podemos situar este modelo en el polo opuesto del anterior. Efectivamente, en este caso se trata de un tipo ideal caracterizado por una simetrización de los roles familiares. Dicha simetrización es justificada en virtud de una con- 
«Papers»: Revista de Sociologia

cepción igualitaria de los individuos, independientemente de su sexo. De esta forma, aunque se admita que biológicamente el hombre y la mujer presentan evidentes diferencias, su proyección social adquiere un rango similar. Bajo este punto de vista no es difícil pensar que en el ámbito familiar se produzca una división del trabajo doméstico no en función del sexo, sino en virtud de otros criterios que las parejas consideran pertinentes.

De forma muy sintética podemos decir que este modelo se caracteriza básicamente por los siguientes rasgos:

1) Democratización interna de la vida familiar que lleva aparejada

2) una distribución racional de las cargas domésticas que, aparentemente, al menos,

3) se organizan en función de criterios como la disponibilidad de tiempo de los cónyuges debido a que

4) tanto hombre como mujer aportan ingresos a la familia en régimen de igualdad o, por lo menos, así son considerados, lo cual tiene como consecuencia que

5) se pueda hablar de un poder difuso, compartido y consensuado que pone de manifiesto

6) la inexistencia aparente de una figura central de autoridad en función del sexo.

7) Todo ello aderezado con una red social laxa facilitada por el hecho de que ambos cónyuges/compañeros trabajan fuera del hogar, lo que contribuye a acrecentar el número de amistades no comunes.

8) El trabajo de la mujer es considerado altamente positivo, por lo que supone de independencia económica, y es la consecuencia lógica de la visión simétrica que se tiene del papel de hombres y mujeres en la familia.

\section{MODELO MIXTO}

Si los dos modelos caracterizados anteriormente parecían ser opuestos, el presente es un híbrido que participa de los componentes con que habíamos definido los otros dos.

Efectivamente, se trata de un tipo ideal en el que permanecen rasgos del modelo tradicional combinados con cierta dosis de rupturismo. La gama existente de posibilidades es variada por lo que aquí nos limitaremos a dibujar someramente los trazos más interesantes.

El discurso que sustenta esta modalidad familiar acepta una división funcional de los roles en virtud del sexo, pero al mismo tiempo se produce una continua y diversa invasión de competencias que en virtud del modelo tradi- 
cional se asignaban de forma segmentada al hombre o a la mujer. No es de extrañar, pues, que algunos autores comiencen a hablar de crisis del modelo de varón haciendo referencia a esta situación.

Dicha invasión de competencias

1) se centra en una participación relativa del varón en las tareas domésticas (de forma coordinada o dirigida por la mujer) y una progresiva introducción de la mujer en ámbitos de poder y decisión reservados tradicionalmente al varón, y

2) se justifica - por la parte femenina - apelando a una relativa independencia económica que facilita el trabajo remunerado de la mujer. No obstante, la calidad de esos ingresos (en ocasiones escasos y habitualmente irregulares, por la ubicación de la mujer casada en el mercado de trabajo sumergido) hace que éstos sean considerados como una ayuda a la fuente principal de ingresos: la del varón. Ello incide en el hecho de que sea éste, en situaciones límite y con una fuerte dosis consensuadora y negociadora, el que acabe imponiendo sus criterios, lo cual facilita la aparición de fuertes conflictos intemos en la familia, sobre todo entre los cónyuges/compañeros.

Por otra parte, la progxesiva asunción de responsabilidades domésticas del varón no es óbice para que la mujer tenga que hacer frente a lo que conocemos como la doble jornada laboral. Se acepta que la mujer trabaje, pero si continúa manteniendo su preeminencia en las tareas domésticas, situación que es aceptada o no por la esposa en función del grado de convencimiento de que su principal tarea reside en el hogar. 
«Papers»: Revista de Sociologia

\section{BIBLIOGRAFÍA}

Alabart et al. (1988). Els rols en el matrimoni i la cohabitació: un estudi a l'àrea de Barcelona, Papers, 30: 139-159.

Alabart, A. \& Solsona, M. (1987), De l'activitat de la dona a Catalunya des de 1970, Barcelona (ejemplar mecanografiado).

Alberdi, I. (1982). Un nuevo modelo de familia, Papers, 18: 87-113.

Blood, R.O \& Wolfe, D.H. (1960), Husbands and wives: the dynamics of married living, Nueva York, The Free Press, citado en Kellerhals (1984), J. Microsociologie de la famille, París, Presses Universitaires de France, pág. 45.

Bott, E. (1981). Family and social network, Londres, Tavistock, (2 edición).

Casas Baamonde, M. E. (1989). Las nuevas formas de empleo en el derecho del trabajo español: evolución y tendencias. Barcelona, Primeres Jornades Interuniversitàries Barcelona-Tolouse, (ejemplar mecanografiado), febrero-marzo.

Casas, J.I. (1987). La participación laboral de la mujer en España, Madrid, Instituto de la Mujer (Ministerio de Cultura), pág. 141.

Edgell, L. (1980). Middle class couples, Londres, Allen \& Unwin.

Engels, F. (1983). El lorigen de la familia, la propiedad privada y el estado, Madrid, Ed. Sarpe.

Flaquer, L. \& Soler, J. (1988). La familia española en transición, mecanografiado, en prensa (por atención de los autores), Barcelona.

Izquierdo, M.J. (1988). Les dones: una forma de desigualtat social, Barcelona, Institut d'Estudis Metropolitans.

Michel, A. (1974). Sociología de la familia y del matrimonio, Barcelona, Ed. Península.

Michel, A. (1974). Activité professionnelle de la femme et vie conjugale, París, Centre National de la Recherche Scientifique.

Ministerio de Trabajo y Seguridad Social (1988). Modalidades de contratación, Madrid, MTSS.

OCDE (1986). Flexibilidad en el mercado de trabajo, Madrid, MTSS.

Piore, M. (1983). Notas para la estratificación del mercado de trabajo, Toharia, L. (comp.) El mercado de trabajo: teorías y aplicaciones, Madrid, Alianza Editorial, pp: 193-223.

Rapoport, R. \& Rapoport, R. (1973). Une famille, denx carrières, París, Ed. Denoël-Gouthicr.

Zufiaur, J.M. (1986). Entre el despido libre y la libertad de despido, en El

País, 15 de septiembre. 